\title{
Awareness and Health Care Practice of Mothers' About Obstetric Danger Signs at Haramaya District, Eastern Ethiopia
}

\author{
Tesfaye Assebe Yadeta ${ }^{1}$, Fekede Asefa Kumsa ${ }^{2}$ \\ ${ }^{1}$ School of Nursing and Midwifery, College of Health and Medical Sciences, Haramaya University, Harar, Ethiopia \\ ${ }^{2}$ Department of Public Health, College of Health and Medical Sciences, Haramaya University, Harar, Ethiopia
}

Email address:

tesfaye.assebe@yahoo.com (T. A. Yadeta), sinboona@gmail.com (F. A. Kumsa)

\section{To cite this article:}

Tesfaye Assebe Yadeta, Fekede Asefa Kumsa. Awareness and Health Care Practice of Mothers' About Obstetric Danger Signs at Haramaya District, Eastern Ethiopia. Journal of Family Medicine and Health Care. Vol. 3, No. 1, 2017, pp. 23-29. doi: 10.11648/j.jfmhc.20170301.15

Received: March 5, 2017; Accepted: March 17, 2017; Published: April 18, 2017

\begin{abstract}
Mother's awareness of potential obstetric danger signs during pregnancy, child birth and immediate post-delivery are crucial to influence their decisions to seek immediate health care. However, their awareness and health care practice were not well known in Haramaya district. Thus, this study was to assess mother's awareness of obstetric danger signs and their health care seeking practice. Community based cross sectional study was conducted in June, 2012 on 757 mothers who gave birth for the last two years and reside in Haramaya District. Sample size was estimated by using a single population proportions formula. Multistage sampling method was used to select study subjects. Data was collected by interview using structured questionnaires. Bivariate and multivariate were used to analyze predictors. Results: Only $86(11.3 \%)$ of respondents mentioned three and above key obstetric danger signs during pregnancy. About half of 402(53\%) of respondents did not aware for at least one obstetric danger signs. 234 (31\%) respondents were reported to have experienced one or more key danger signs but $31.2 \%$ of them did not sought for medical treatment. Mothers who followed primary education 2.7 times [AOR 2.7; 95\% CI 1.5-7.2], and secondary and above education 2.4 times [AOR 2.4; 95\% CI 1.3-5.4] more likely to have awareness on obstetric danger signs during pregnancy as compared to those didn't attained formal education. Mothers who attended antenatal care were 4.28 times [AOR 4.28; 95\% CI 2.01-9.12] more likely of having awareness on obstetric danger sign than mother who did not attended ANC. Similarly, the odds of giving birth at health institutions increased the level of awareness about obstetric danger signs about three fold [AOR 3.23; 95\% CI 1.821-5.742] than those who giving birth at home. Conclusion: Awareness of mothers on obstetric danger signs in the study area and health seeking practice were low. Thus, community education and information during antenatal Care utilization, institutional delivery services and postnatal care about obstetric danger signs and its consequence if the mother will not seeking care are important steps in improving the awareness and health seeking practice.
\end{abstract}

Keywords: Antenatal Care, Awareness Maternal Health, Obstetric Danger Signs

\section{Introduction}

Maternal and newborn mortality remains a major public health challenge in developing countries, and little progress made towards the achievement of millennium development goals [1]. The Maternal mortality ratio (MMR) in developing regions 240/100,000 live birth was 15 times higher compared to developed regions. Sub- Saharan Africa has the highest MMR at 500 maternal deaths per 100000 live births [2]. In Ethiopia, 676 per 100000 live birth maternal mortality is among the highest in the world [3].

The history of successes in reducing maternal and newborn mortalities showed that skilled professional care during and after childbirth can make the difference between life and death for both women and their newborn babies [4]. Sharp decline in maternal mortality rates after 1930s in developed country were due to standard of maternal care provided by skilled birth attendants [5, 6] Evaluation of safe motherhood initiative program in 1997 also concluded that a skilled attendant to assist childbirth is the single most critical 
intervention to reduce maternal mortality [7].

Obstetric danger signs life-threatening and serious conditions related to pregnancy, if not treated on time it could results in loss of mothers and the babies. These key danger signs during pregnancy, include severe vaginal bleeding, swollen hands/face and blurred vision. Key danger signs during labor and childbirth include severe vaginal bleeding, pro-longed labor, convulsions, and retained placenta. Key danger signs during the postpartum period include severe bleeding; foul smell vaginal discharge and fever. These life threatening complications are treatable and avoidable, if women with the complications can identify the risk can be averted [8].

Factors that affect maternal and neonatal mortality and morbidity are generalized in to three delays: delays in deciding to seek care, delays in reaching care, and delays in receiving care. These delays have many causes [9]. Awareness about the significance of symptoms and signs of obstetrics complications may lead to timely access to appropriate emergency obstetric care and crucial to prevents the first and second delay [8].

Therefore, women's awareness of potential danger signs of pregnancy, childbirth and the postpartum period is crucial to influence their decisions to have immediate care seeking taken place, followed by immediate medical attention which improve early detection of problems and reduces the delay in deciding to seek obstetric care [10-12].

The government of Ethiopia (GOE) in 2006 planned to ensure that $80 \%$ of all families recognize important obstetric danger signs related to pregnancy in areas where health extension program is fully implemented; to increase the proportion of births attended by skilled health personnel from 6 percent to 60 percent; Increase national antenatal care coverage levels from 28 percent to 70 percent; [13]. However, Ethiopia continues to score poor record of WHO recommended four and more antenatal care visit (19\%) and delivery at a health facility by skilled health providers $(10 \%)$ in 2011[3]. Even though about $80 \%$ maternal and neonatal death occurs during childbirth and within the first week after child birth, only 7 percent of women received postnatal care in the first two days $[3,4,14]$.

The study conducted in Northern part of Ethiopia also revealed, a small proportion $10.9 \%, 2.2 \%$ and $5.2 \%$ of the respondents had knew vaginal bleeding, blurred vision and swollen hands/face key danger signs during pregnancy, respectively. Key danger signs during labor/childbirth $16.5 \%$, $11 \%, 7.1 \%$ and $0.6 \%$, of the respondents spontaneously mentioned severe vaginal bleeding, prolonged labor, retained placenta and convulsions as danger signs respectively. $16.7 \%, 1.1 \%$ and $1.5 \%$ of the respondents spontaneously mentioned severe vaginal bleeding, high fever, and foul smelling vaginal discharge as danger signs during postpartum period, respectively [15].

The gap between the planned and expected in awareness on danger sign and maternal health care seeking practice in the country need investigation. Therefore this research assesses of mother's awareness on obstetric danger signs and their health care seeking practice when they experienced obstetric danger sign.

\section{Materials and Methods}

\subsection{Study Area and Period}

The study was conducted in Haramaya district, East Hararge, Oromia Region, Eastern part of Ethiopia which is located $507 \mathrm{~km}$ East of Addis Ababa in June, 2012.

\subsection{Study Design}

community based cross sectional study was conducted.

\subsection{Study Populations}

All mothers who gave birth for the last two years and reside in Haramaya district were source populations. Mothers who randomly selected were study populations.

\subsection{Sample Size Determination and Sampling Techniques}

Sample size was estimated by using single population proportions formula, we made the following assumptions: proportion of mother awareness of obstetric danger $\operatorname{sign}(\mathrm{p}=0.36)[16], 5 \%$ marginal of error, $95 \%$ confidence level, design effect of two and by considering $10 \%$ nonresponse rate the final sample size was 779 . Multistage sampling method was used to select study subjects. Four kebeles (the lowest units of administration in Ethiopia) were selected randomly (one from urban and three from rural). Then, women who gave birth in the last two years were reviewed from health extension records in selected kebeles and simple random sampling were used to select the study subject.

\subsection{Operational and Term Definitions}

\subsubsection{Awareness on Key Danger Signs of Pregnancy}

In this research a woman was considered as having awareness if she can mention at least three key danger signs for pregnancy.

\subsubsection{Awareness on Key Danger Signs of Labor/Childbirth}

In this research a woman was considered as having awareness if she can mention at least three key danger signs for Labor/childbirth spontaneously or after prompting.

\subsubsection{Awareness on Key Danger Signs of Postpartum}

In this research a woman was considered having awareness if she can mention at least the three key danger signs for postpartum spontaneously or after prompting

\subsubsection{Experienced of Obstetric Danger Sign(s)}

Any sign of obstetric danger sign(s) reported by woman which may occur in women during pregnancy, delivery or within 6 weeks after delivery.

\subsection{Data Collection}

A safe motherhood questionnaire developed by the 
Maternal and Neonatal Program of JHPIEGO, an affiliate of John Hopkins University was used [8]. The questionnaires consist of awareness about obstetric danger sign, sociodemographic data such as age, educational status, occupation, family size; reproductive health characteristics such as gravidity, parity and antenatal follow up, health care practice and reason for not seeking care. Eight Diploma holders midwife/nurse female data collectors and two Bachelor of Science degrees (BSc) holder health professionals fluent to local language Afaan Oromo were recruited. The data was collected by face to face interview.

\subsection{Data Quality Control}

Questionnaire for data collection was prepared in English and translated to Afan Oromo. Its consistency checked by re translating back to English. Training was given for two days for data collectors and supervisors about study objectives and data collection procedures. Data collection procedures were supervised by supervisors and principal investigator and checked for completeness and coherence at the site of data collection.

\subsection{Data Processing and Analysis}

The data was coded, entered in to computer and cleaned using Epi-info version 3.5.1 and transported to SPSS version 16 for analysis. First descriptive statistics and binary logistic regression was used to assess the association of mothers awareness about obstetric danger signs with exploratory variables. Factors for which significant $(\mathrm{P} \leq 0.2)$ bivariate association observed were retained for subsequent multivariate analyses using logistic regressions. Adjusted odds ratios (OR) with $95 \%$ confidence intervals (CI) were calculated.

\subsection{Ethical Consideration}

Before commencement of data collection, ethical approval was obtained from the Institutional Research and Ethical Review Committee (IRERC) of College of Health and Medical Science, Haramaya University. College of Health and Medical Science sent an official letter to Haramaya district health bureau. Then at the time of interview, informed written consent of all study participants was obtained.

\section{Results}

\subsection{Socio-Demographic Characteristics}

A total of 757 mothers responded to the questionnaire, this gives a response rate $97.2 \%$. The age of respondents ranged from 16 to 40 years (mean $\pm \mathrm{SD}=24.6 \pm 4.3$ years). Almost all respondents were Muslim (97.5\%), and Oromo (95.6\%) ethnicity. Nearly three quarter $(74 \%)$ of the respondents had not attended formal education. The numbers of children respondents have ranged from 1 to $11(3.3 \pm 1.9)$ (Table 1).
Table 1. Socio-Demographic characteristics mother at haramaya district, Eastern Ethiopia, June 2012.

\begin{tabular}{|c|c|c|}
\hline Characteristics & numbers & percentages \\
\hline \multicolumn{3}{|l|}{ Age of respondent } \\
\hline $16-25$ & 507 & 67.0 \\
\hline $26-35$ & 226 & 29.8 \\
\hline$>35$ & 24 & 3.2 \\
\hline \multicolumn{3}{|l|}{ Residence } \\
\hline Urban & 197 & 26.03 \\
\hline Rural & 560 & 74.97 \\
\hline \multicolumn{3}{|l|}{ Religion } \\
\hline Muslim & 738 & 97.5 \\
\hline Christian & 19 & 2.5 \\
\hline \multicolumn{3}{|l|}{ Ethnic group } \\
\hline Oromo & 724 & 95.6 \\
\hline Amara & 15 & 2.0 \\
\hline Others* & 18 & 2.4 \\
\hline \multicolumn{3}{|l|}{ Marital status } \\
\hline Married & 707 & 93.4 \\
\hline Divorced & 23 & 3.1 \\
\hline Widowed & 14 & 1.8 \\
\hline Separated & 13 & 1.7 \\
\hline \multicolumn{3}{|l|}{ Educational status } \\
\hline No formal education & 560 & 74.0 \\
\hline Primary & 148 & 19.6 \\
\hline Secondary and above & 49 & 6.4 \\
\hline \multicolumn{3}{|l|}{ Family size } \\
\hline $1-5$ & 448 & 59.2 \\
\hline $6-10$ & 293 & 38.7 \\
\hline$>10$ & 16 & 2.1 \\
\hline \multicolumn{3}{|l|}{ Number of children } \\
\hline $1-3$ & 140 & 18.5 \\
\hline $4-5$ & 313 & 41.3 \\
\hline$>5$ & 304 & 40.2 \\
\hline
\end{tabular}

\subsection{Reproductive Health Characteristics}

Mothers, 323 (42.7\%) had ANC follow up at least once for the most recent pregnancy. $23.9 \%$ of them attended four and above ANC visit by then, only about one tenth mothers $13.2 \%$ birth at health institutions (Table 2).

Table 2. A reproductive health characteristic of Women's at Haramaya district, Eastern Ethiopia, June 2012.

\begin{tabular}{lll}
\hline Characteristics & number & percentage \\
\hline Parity & 530 & 70.0 \\
$1-4$ & 227 & 30.0 \\
$\geq 5$ & 552 & 72.9 \\
Gravidity & 205 & 27.1 \\
$1-4$ & & \\
$\geq 5$ & 323 & 42.7 \\
ANC visits & 434 & 57.3 \\
Yes & & \\
No & 246 & 76.1 \\
Number of ANC visit & 77 & 23.9 \\
Partially followed & & \\
Fully followed & 245 & 31 \\
Presence of complications & 512 & 69 \\
Yes & & \\
No & 657 & 86.8 \\
Place of delivery & 100 & 13.2 \\
Home & & 95.7 \\
Health institutions & 725 & 1.9 \\
Modes of delivery & 14 & 2.4 \\
Spontaneous vaginal delivery & 18 & \\
Cesarean section & & \\
Instrumental delivery & & \\
\hline
\end{tabular}




\subsection{Mothers' Awareness of Key Obstetric Danger Signs}

Awareness of three or more key obstetric danger signs during pregnancy, delivery and postpartum were $11.36 \%$,
$13.08 \%$ and $14.53 \%$, respectively. Moreover, $53 \%, 52.2 \%$ and $47.2 \%$ of respondents didn't have awareness for at least one obstetric danger signs during pregnancy, delivery and postpartum respectively (Table 3 ).

Table 3. Characteristics of study participants by knowledge of danger sign, Haramaya District, 2012.

\begin{tabular}{|c|c|c|c|c|c|c|}
\hline \multirow[t]{2}{*}{ characteristics } & \multicolumn{2}{|c|}{$\begin{array}{l}\text { Danger sign Awareness during } \\
\text { pregnancy }\end{array}$} & \multicolumn{2}{|c|}{ Danger sign Awareness during delivery } & \multicolumn{2}{|c|}{ Danger sign Awareness during post natal } \\
\hline & yes & no & yes & no & yes & no \\
\hline \multicolumn{7}{|l|}{ Age } \\
\hline $15-25$ & 53 & 472 & 47 & 478 & 71 & 454 \\
\hline $26-35$ & 30 & 190 & 49 & 171 & 33 & 187 \\
\hline$>35$ & 3 & 9 & 3 & 9 & 6 & 6 \\
\hline \multicolumn{7}{|l|}{ Educational status } \\
\hline No formal education & 43 & 520 & 43 & 520 & 71 & 492 \\
\hline Primary $(1-8)$ & 25 & 120 & 39 & 106 & 19 & 126 \\
\hline $\begin{array}{l}\text { Secondary and above } \\
\text { marital }\end{array}$ & 18 & 31 & 17 & 32 & 20 & 29 \\
\hline married & 69 & 635 & 78 & 626 & 103 & 601 \\
\hline \multicolumn{6}{|l|}{ number of children } & 46 \\
\hline $1-3$ & 65 & 393 & 64 & 394 & 59 & 399 \\
\hline $4-6$ & 18 & 230 & 33 & 215 & 44 & 204 \\
\hline $\begin{array}{l}7 \text { and above } \\
\text { religion }\end{array}$ & 3 & 48 & 2 & 49 & 7 & 44 \\
\hline Muslim & 76 & 662 & 89 & 649 & 101 & 637 \\
\hline $\begin{array}{l}\text { Christian } \\
\text { parity }\end{array}$ & 10 & 9 & 10 & 9 & 9 & 10 \\
\hline $1-3$ & 56 & 357 & 56 & 357 & 52 & 361 \\
\hline $4-6$ & 26 & 235 & 31 & 230 & 37 & 224 \\
\hline$>6$ & 4 & 79 & 12 & 71 & 21 & 62 \\
\hline \multicolumn{7}{|l|}{ ANC visit } \\
\hline No & 16 & 418 & 17 & 417 & 20 & 414 \\
\hline yes & 70 & 253 & 82 & 241 & 90 & 233 \\
\hline \multicolumn{7}{|l|}{ Place of delivery } \\
\hline Home & 40 & 534 & 37 & 537 & 54 & 520 \\
\hline Health institution & 46 & 137 & 62 & 121 & 56 & 127 \\
\hline \multicolumn{7}{|l|}{ Mode of delivery } \\
\hline Normal & 78 & 647 & 91 & 634 & 101 & 624 \\
\hline $\mathrm{CS}$ & 7 & 7 & 7 & 7 & 8 & 6 \\
\hline Assisted by instrument & 1 & 17 & 1 & 17 & 1 & 17 \\
\hline
\end{tabular}

\section{Mothers' Awareness on Specific Key Obstetric Danger Signs}

The most frequently mentioned key danger signs was severe vaginal bleeding; during pregnancy $23.5 \%$, delivery $29.8 \%$ and postpartum period $32.7 \%$ (Table 4 ).

Table 4. Proportion of Women's awareness on key obstetric danger signs at Haramaya district, Eastern Ethiopia, June 2012 (N=757).

\begin{tabular}{lllllll}
\hline \multirow{3}{*}{ Obstetric danger sign } & \multicolumn{6}{l}{ Awareness of danger signs } \\
\cline { 2 - 7 } & \multicolumn{1}{l}{ Pregnancy } & delivery & after delivery \\
\cline { 2 - 7 } & $\mathbf{n}$ & $\mathbf{\%}$ & $\mathbf{n}$ & $\mathbf{\%}$ & $\mathbf{n}$ & $\mathbf{\%}$ \\
\hline vaginal bleeding & 178 & 23.5 & 232 & 29.8 & 255 & 32.7 \\
convulsion & 84 & 11 & 121 & 15.5 & 104 & 13.4 \\
Sever head ache & 97 & 12.5 & & & 131 & 16.8 \\
Blurred vision & 88 & 11.6 & & & 34 & 4.4 \\
Hand/face edema & 26 & 3.3 & & & & \\
retained placenta & & & 98 & 12.8 & & \\
prolonged labour & & & 188 & 24.1 & & \\
Offensive vaginal discharge & & & & & 25 & 3.2 \\
high grade fever & 115 & 14.8 & & & 231 & 29.7 \\
others* & 74 & 9.7 & 58 & 7.7 & 38 & 5.02 \\
I don't know & 402 & 53 & 395 & 52.2 & 357 & 47.2 \\
\hline
\end{tabular}

*multiple response applicable.

\subsection{Factors Associated with Mothers' Awareness on Obstetric Danger Signs}

As compared to mothers who did not attained formal education, those mothers who followed primary education were 2.99 times and secondary and above education 2.38 times more likely aware on obstetric danger signs during pregnancy [AOR 2.99; 95\% CI (1.264-7.074)], and [AOR $2.38 ; 95 \%$ CI 1.025-5.551], respectively. Mothers those who attend ANC during their pregnancy were 4.28 times, 5.72 times and 6.42 times more likely to aware of obstetric danger signs during pregnancy, child birth, and postpartum period compared to mothers who did not attended ANC [AOR 4.28; 95\% CI 2.01-9.12], [AOR 5.72; 95\% CI 3.05-10.71], and [AOR 6.42; 95\% CI 3.67-11.21], respectively. In addition, As compared to women who did not giving birth at health institutions during their pregnancy, those who giving birth at health institutions were 3.23 times, 2.43 times and 2.38 times more likely to aware of obstetric danger signs during pregnancy, delivery and postpartum period [AOR 3.23; 95\% CI 1.82-5.72], [AOR 2.43; 95\% CI 1.33-4.44], [AOR 2.38; 
95\% CI 1.34-4.22], respectively (Table 5).

Table 5. Factors associated with mothers' awareness on obstetric danger sign at Haramaya district, Eastern Ethiopia, June 2012.

\begin{tabular}{|c|c|c|c|}
\hline Characteristic & $\begin{array}{l}\text { AOR }(95 \% \text { CI }) \\
\text { Pregnancy danger sign Awareness }\end{array}$ & $\begin{array}{l}\text { AOR }(95 \% \text { CI) } \\
\text { Delivery danger sign awareness }\end{array}$ & $\begin{array}{l}\text { AOR }(95 \% \text { CI) } \\
\text { After delivery danger sign awareness }\end{array}$ \\
\hline \multicolumn{4}{|l|}{ Marital status } \\
\hline Married & 1 & 1 & 1 \\
\hline Others* & $4.219(1.889-9.423)^{*}$ & $4.163(1.888-9.177)^{*}$ & $.469(.182-1.206)$ \\
\hline \multicolumn{4}{|l|}{ Educational status } \\
\hline No formal education & 1 & 1 & 1 \\
\hline Primary & $2.990(1.264-7.074)^{*}$ & $1.462(.596-3.587)$ & $1.270(.535-3.014)$ \\
\hline Secondary and above & $2.385(1.025-5.551)^{*}$ & $.476(.196-1.152)$ & $1.600(.654-3.913)$ \\
\hline \multicolumn{4}{|l|}{ Gravidity } \\
\hline $1-2$ & $.261(.083-.816)$ & $1.148(.506-2.606)$ & $3.262(1.658-6.419) *$ \\
\hline $3-4$ & $.368(.115-1.172)$ & $1.074(.458-2.522)$ & $1.949(.982-3.868)$ \\
\hline 5 and above & 1 & 1 & 1 \\
\hline \multicolumn{4}{|l|}{ ANC visit } \\
\hline No & 1 & & \\
\hline Yes & $4.285(2.013-9.123)^{*}$ & $5.721(3.053-10.719)^{*}$ & $6.418(3.674-11.211)^{*}$ \\
\hline \multicolumn{4}{|l|}{ Place of delivery } \\
\hline Home & 1 & 1 & 1 \\
\hline Health institution & $3.234(1.821-5.742)^{*}$ & $2.437(1.335-4.449)^{*}$ & $2.382(1.344-4.223)^{*}$ \\
\hline \multicolumn{4}{|l|}{ Mode of delivery } \\
\hline SVD & 1 & 1 & 1 \\
\hline $\mathrm{CS}$ and instrumental & $8.2(1.2-20.4)^{*}$ & $27.9(6.5-32.6)^{*}$ & $7.2(0.7-18.3)$ \\
\hline
\end{tabular}

$*=\mathrm{p}<0.05 * *$ widowed, divorced, separated SVD spontaneous vaginal delivery, CS cesarean section

\subsection{Proportions of Mothers Experienced Obstetric Danger Signs}

Two hundred thirty four (234) respondents were reported to have faced one or more key danger signs. Key danger signs faced were $30(13 \%), 115(49.2 \%)$ and $89(37.8 \%)$ during the most recent pregnancy, child birth and postpartum period respectively. Of 30 women who faced problem during pregnancy, $11(36.7 \%)$ faced high grade fever, $7(23.3 \%)$ experienced vaginal bleeding and the rest $12(40 \%)$ faced others danger signs like; severe head ache, face swelling and severe abdominal pain. Out of 115 mothers who faced delivery related problem $57(49.6 \%)$ faced prolonged labor, $26(22.6 \%)$ faced retained placenta and the rest $32(27.8 \%)$ face other dangers signs like severe vaginal bleeding and convulsion. Of 89 mothers who faced problem during postpartum period 31(34.8) faced high grade fever, $27(30.3 \%)$ breast pain, $18(20.3 \%)$ and the rest $13(14.6 \%)$ faced other dander signs.

\subsection{Health Care Seeking Practice and Reason Mentioned for not Seeking Care}

Of mothers who experienced complication of which 159 $(68.8 \%)$ were sought treatment at health institutions, 58 $(25.1 \%)$ were not treated or stayed at home and the rest 14 $(6.1 \%)$ went to cultural or religious healers. As shown in figure 1, out of 72 mothers, who were not sought for medical treatment, $21(29.3 \%)$ were due to lack of money while $16(22.2 \%)$ were due to perceiving that problem was harmless.

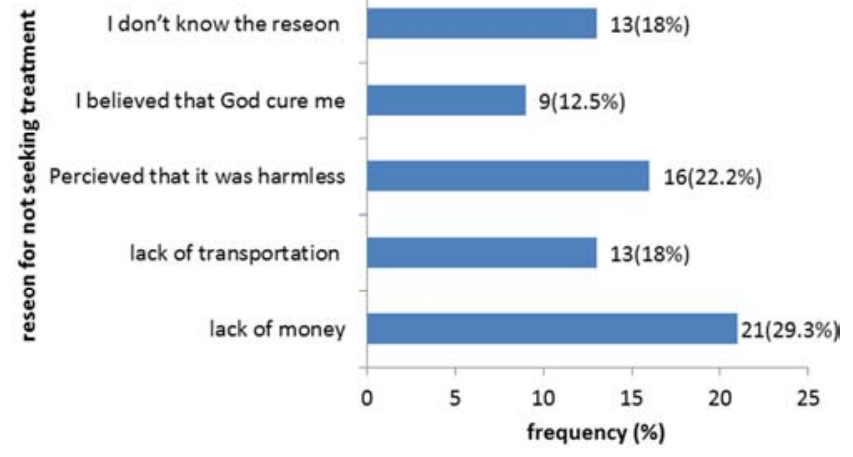

Figure 1. Reseons of mothers not seeking medical treatment for experanced obstatric danger signs, at Haramaya district, Eastern Ethiopia, June 2012 $(N=72)$.

\section{Discussion}

Mothers' awareness of obstetric danger signs was low. Having ANC follow up on the most recent pregnancy, giving birth at health institution, maternal educational status and giving recent birth by caesarian section and instrumental delivery were associated with higher likelihood of mothers' good awareness on obstetric danger signs. Significant numbers of mothers did not sought for treatment obstetric complications. Financial obstacles, lack transportation and perceiving that problem was harmless were the major reasons for not seeking medical treatments.

Awareness of mothers on danger sign in this study was very low. Less than $15 \%$ of the mothers mentioned three and more key obstetric danger signs. Almost half of the women were not aware of at least one obstetric danger sign. Low awareness to maternal danger sign may also contribute for maternal health care utilization which is very low in the 
country. Because, women's awareness of potential danger signs is crucial to influence their decisions to have immediate obstetric care, and reduces the delay in deciding to seek obstetric care $[11,12]$. This finding is similar with study in Tanzania [17]. Similar finding also reported from Egypt [18]. Lower than study in Uganda (18.7\%) mentioned three and above key danger signs [19] and in Ghana, 86.7\% [20] of mothers mentioned at least one obstetric danger sign. These differences in awareness level could be due to a difference in socio demographic, cultural, and health interventions.

Vaginal bleeding was the most recognized obstetric danger sign, and was mentioned by $23.5 \%, 29.8 \%$ and $32.7 \%$ of respondents during pregnancy, delivery and after delivery respectively. Excessive vaginal bleeding and prolonged labor were easily identified, severe and frequently occurs. This may contribute most commonly recognized danger sign. Similar to our study, higher awareness of vaginal bleeding were reported by different studies [19, 21, 22]. Additionally, about $24 \%$ of mothers aware of prolonged labor as obstetric danger sign and it was the most recognized danger sign during delivery as reported in Haiti [23].

In this study educational status was found to associate with awareness on obstetric danger signs during pregnancy. Even though education enhance awareness of danger sign, more than three quarter of mother's were not attending any formal education and only few of them attend secondary and above level in the study area that hence, attention should be given for illiterate pregnant women while to address their specific needs. Higher level of education was associated with increased awareness of obstetric danger signs. Similar finding was reported from different studies [17, 18, 24]. However, some studies reported contradictory finding [16].

In this study, $42.7 \%$ of mothers were having at least once ANC visit. This is much less than study in Uganda (68\%) [19], Sudan (83.3\%) [25], Tanzania (94\%) [26] and Ghana (95\%) [20] of mothers had at least once ANC visit. And, only $10 \%$ of the mothers fully followed ANC as per WHO recommendation but $16.2 \%$ in Sudan[25] and $62.1 \%$ in Nepal [27]. Having ANC follow up for the last recent pregnancy increased the likelihood of good awareness on Obstetric danger signs by 4.28 folds, 5.72 folds and 6.41 folds during pregnancy, delivery and after delivery respectively. Similarly, different study reported ANC attendance increases mothers' awareness of obstetric danger signs [28, 29]. In addition having four or more ANC visit increases the odds of mothers' awareness on obstetric danger signs. This finding is consistent with another study [21]. This might be because ANC provide opportunity to educate women and their family about danger signs related to pregnancy [30]. Evidences also revealed that information obtained during effective implementation of ANC enhances the awareness of maternal danger signs [10, 16, 21].

Only $13.2 \%$ of the respondent gave the last recent birth at health institutions. This very low and even incomparable with other African countries: $99.8 \%$ in Ghana[20], 97\% in Kinshasa[31] and 57.2\% in Kenya [32]. Giving birth at health institution was associated with increased likelihood of good awareness on obstetric danger signs. Moreover, mothers who gave birth by caesarian section and instrumental delivery were more aware of obstetric danger signs than who gave birth by spontaneous vaginal delivery. This might be due to mothers gave birth by caesarian section and instrumental delivery when they faced obstetric complication. Therefore, they easily remembered the problem they faced. Facing pregnancy related complication for the last pregnancy increases the likelihood of good awareness of obstetric danger signs. Study in Egypt also indicated similar finding [18].

Out of mothers who faced obstetric complication, significant numbers of mothers (31.2\%) were not sought for medical treatment. This is might be due to the awareness of mothers on obstetric danger signs in the study area is low. The reasons raised were financial obstacles, lack transportation and perceiving that problem was harmless. This is consistent with another study [33].

\section{Conclusions}

Awareness of mothers on obstetric danger signs in the study area is low. Mothers who had primary and secondary education have more likely of awareness on obstetric danger sign during pregnancy than those who did not attended any formal education. In addition, having ANC follow up, and giving birth at health institution were associated with higher likelihood of mothers' awareness of obstetric danger signs. Significant numbers of mothers were not sought for medical treatment when they experienced obstetric complications. Financial obstacles, lack of transportation and perceiving that problem was harmless were the major barriers for not seeking medical treatments. Thus, community education and information during antenatal Care utilization, institutional delivery services and postnatal care about obstetric danger signs and its consequence if the mother will not seeking care are important steps in improving the awareness and health seeking practice.

\section{Competing Interest}

The author(s) declare that they have no competing interests.

\section{Authors' Contribution}

TA has conceived the study, carried out the overall design and execution of the study, performed data collection, performed statistical analysis and involved in draft of the manuscript. FA has critically revised the design, performed statistical analysis and drafted the manuscript. Both authors read and confirmed the manuscript for submission.

\section{Acknowledgments}

The authors would like to thank Haramaya University for funding this study as part of its young faculty research grant. 


\section{References}

[1] WHO, Trends in maternal mortality: 1990 to 2010. 2012, WHO Geneva 27, Switzerland.

[2] UN, The Millennium Development Goals Report. 2013, UN: Newyork.

[3] Central Statistical Agency, Ethiopia Demographic and Health Survey 2011 Central Statistical Agency Addis Ababa,Ethiopia.

[4] World Health Organization (WHO), The World Health Report 2005 Make every mother and child count. 2005, WHO: Geneva.

[5] Loudon. I, Maternal mortality in the past and its relevance to developing countries today. The Americal Journal of Clinical Nutrition 2000;. 72.

[6] Chamberlain. G, British maternal mortality in the 19th and early 20th centuries. Journal of the Royals Socity of Medicine, 200699.

[7] UNFPA., Evaluation Finding. 1999: New York 220 East 42nd Street.

[8] JHPIEGO, Monitoring birth preparedness and complication readiness: tools and Indicators for maternal and newborn health. 2004, John hopking unversity.

[9] Thaddeus S. and M. D, Too far to walk: maternal mortality in context.. Soc Sci Med, 1994. 38(1091): p. 110.

[10] Jammeh, A., J. Sundby, and S. Vangen, Barriers to Emergency Obstetric Care Services in Perinatal Deaths in Rural Gambia ISRN Obstetrics and Gynecology, 2011.

[11] Warren, C., Care seeking for maternal health: challenges remain for poor Women Ethiop. J. Health Dev., 2010. 24(Special Issue 1): p. 100-104.

[12] Michael, K. A., et al., Maternal Health and Care-Seeking Behavior In Bangladesh: Findings from a National Survey International Family Planning Perspectives. 2007.

[13] Federal Democratic Republic of Ethiopia, National Reproductive Strategy, 2006-2015, FMOH, Editor. 2006, Ministry of Health: Addis Ababa.

[14] WHO, Ditrict planning tool for maternal and newborn strategy implimentation. A. practicel tool for strengthening health managment system in making pregnancy safe. 2011, WHO: Geneva.

[15] Hiluf, M. and F. M, Birth Preparedness and Complication Readiness among women in Adigrat town. Ethiopian Journal of. Health Development, 2007. 22(1): p. 14-20.

[16] Hailu, M., A. Gebremariam, and F. Alemseged, knowledge about obstetric danger signs among pregnant women in aleta wondo district, sidama zone, southern Ethiopia. Ethiop J Health Science, 2010. 20(1).

[17] Urassa, D. P., A. B. Pembe, and F. Mganga, Birth preparedness and complication readiness among women in Mpwapwa district, Tanzania. Tanzania Journal of Health Research, 2012. 14(1).

[18] Rashad, W. A. and R. M. Essa, Women's Awareness of Danger Signs of Obstetrics Complications Journal of American Science 2010. 6(10).
[19] Jerome K. Kabakyenga, et al., Knowledge of obstetric danger signs and birth preparedness practices among women in rural Uganda. Reproductive Health, 2011. 8(33).

[20] Udofia., E. A., et al., Birth and Emergency Planning: A. Cross Sectional Survey of Postnatal Women at Korle Bu Teaching Hospital, Accra, Ghana African Journal of Reproductive Health, 2013. 17(1): p. 27-40.

[21] Andrea B. Pembe, et al., Rural Tanzanian women's awareness of danger signs of obstetric complications. BMC Pregnancy and Childbirth, 2009. 9(12): p. 1-8.

[22] Hadayat A. Amasha and Manar F. Heeba, Maternal Awareness of Pregnancy Normal and Abnormal Signs: An Exploratory Descriptive Study. Journal of Nursing and Health Science 2013. 2(5): p. 39-45.

[23] Chia-Y. u Kuo, Community Awareness of Maternal and P. erinatal Danger Signs in Rural Haiti. in University of Connecticut Health Center Graduate School. 2006, University of Connecticut. p. 96.

[24] Kabakyenga, J. K., et al., Knowledge of obstetric danger signs and birth preparedness practices among women in rural Uganda. Reproductive Health, 2011. 8(33).

[25] Ali., A. A. A., et al., Awareness of danger signs and nutritional education among pregnant women in Kassala, Eastern Sudan. Sudanese journal of public health, 2010. 5(4): p. $179-181$.

[26] Declare Mushi, Rose Mpembeni, and Albrecht Jahn, Effectiveness of community based safe motherhood promoters in improving the utilization of obstetric care. The case of Mtwara Rural District in Tanzania. BMC Pregnancy and Childbirth, 2010. 10(14): p. 1-9.

[27] Gyawali., K., et al., Knowledge and practices on maternal health care among mothers: A. Cross sectional study from rural areas of mid-western development region Nepal. Journal of the Scientific Society, 2013. 40(1): p. 9-13.

[28] Doctor, H. V., et al., awareness of Critical Danger Signs of Pregnancy and Delivery, Preparations for Delivery, and Utilization of Skilled Birth attendants in nigeria. Journal of Health Care for the Poor and Underserved, 2013. 24: p. 152-170.

[29] Renu Sangal, et al., Knowledge and Practices regarding Obstetric danger signs in Women attending Ante-natal care Clinic at BRD Medical College Gorakhpur. Indian J. prev. soc. Med., 2012. 43(1): p. 12-18.

[30] Nieburg, p., Improving Maternal Mortality and Other Aspects of Women's Health: The United States' Global Role. 2012, Center for strategic \& International studies, Global Health Policy Center: Oslo. p. 1-21.

[31] Kabali E, Gourbin C, and De Brouwere C, Complications of childbirth and maternal deaths in Kinshasa hospitals: testimonies from women and their families BMC Pregnancy and Childbirth, 2011. 11(29).

[32] Wanjira C, et al., Delivery Practices and Associated Factors among mothers seeking Child Welfare Services in Selected Health Facilities in Nyandarua South District, Kenya. BMC Public Health 2011. 11(360).

[33] Lubbock., L. A. and R. B. Stephenson., Utilization of maternal health care services in the department of Matagalpa, Nicaragua. Pan Am J. Public Health, 2008. 24(2): p. 75-84. 